\title{
Bringing a Little Order to the Chaos: From the Operating Room, and Beyond
}

Can J Neurol Sci. 2013; 40: 282-283

So what can you think of that might cause you frustration and lead you to complain today? Please review the list below. If you are a neurosurgeon check off all that apply and feel free to add anything that is missing. If you are not a neurosurgeon please indulge your surgical colleagues and then create a list of your own that is appropriate to your speciality. Go ahead - it's fun to vent.

\section{Neurosurgical frustration list:}

1. What are the chances that my cases will be cancelled today because there are no ward beds?

2. What are the chances that one of my long and difficult cases will be cancelled today because there are no neuroobservation beds and/or intensive care unit beds?

3. What are the chances that one of my cases will get cancelled today because a surgical colleague who is "on-call" will admit a patient with an urgent problem that will "bump" my cases?

4. How will I enjoy explaining these cancellations to my patients, some who drove for many hours to have their surgery and now have to return home frustrated themselves?

5. What are the chances that one of my cases will prove to be more difficult than anticipated and that I will overrun the official end time for the day, forcing nursing staff into overtime (and finding myself with a black mark in the operating room administrative log book)?

6. What are the chances that my cases will run very smoothly and that I will finish them before the official end time for the day, leading to documentation that operating room resources and nursing staff time are being wasted (again finding myself with a black mark in the operating room administrative log book)?

7. What are the chances that I will work with a careful but slow anesthetist and run over time (see 5 above)? What are the chances that I will work with a careful but fast anesthetist and finish early (see 6 above)?

8. What are the chances, that in order to finish my cases exactly on time (see 5-7 above), I will have to do more of the cases myself, depriving the neurosurgery resident/fellow of a valuable learning experience, thus impacting his/her education (and finding myself with a black mark in the residents' virtual (or real!) log book and perhaps affecting adversely my institutions teaching reputation)?

9. Will the medical student (who has waited patiently for hours while we worked under the microscope) get a chance to do any suturing at all during the closure (see 5-8 above)? (if not, adversely impacting his/her experience during the neurosurgical rotation and a possible interest in a neurosurgical career).

10. What if a piece of equipment malfunctions either before or during the case (leading possibly to $4,5,7,8,9$ above)?
Enough venting; we all have problems. What is more important, perhaps, is how we deal with these problems. In this issue of the Canadian Journal of Neurological Sciences an article entitled "Priority Setting in Neurosurgery as Exemplified by an Everyday Challenge"1 suggests a proactive method to address the specific issue of patient cancellations in the operating room. It does this by adapting an existing framework for health care management called "accountability for reasonableness" (abbreviated A4R) which has potential implications for large scale health resource allocation as well as helping to solve daily rationing scenarios ${ }^{2}$.

What leads to these problems and to the varying degrees of chaos associated with them? Chaos theory is based on the study of nonlinear dynamic systems ${ }^{3}$. These systems can exist in different states of equilibrium - stable equilibrium independent of time, periodic stability where the system periodically comes back to its previous state, and chaotic behavior where small changes can have an unexpected and important impact. Nonlinear dynamic systems have been recognized by mathematicians for more than a century and have been studied mainly in the natural sciences. However more recently the chaos theory has been applied to weather patterns, jet engine propulsion, demographic evolution, economic cycles and stock market evolutions. It has been popularized in modern culture in movies such as "Jurassic Park" and the "Butterfly Effect", the latter which takes its name from the famous metaphor of Lorenz in which the flap of a distant butterfly's wing leads to the creation of a hurricane a few months later ${ }^{4,5}$. Now chaos theory is being applied to organizations. Inherent in organizations are the continuous processes of convergence and divergence, stability and instability, evolution and revolution ${ }^{3}$. Hence organizations will exhibit the qualitative properties of chaotic systems. Or put another way: organizations are potentially chaotic; the greater number of counteracting forces in an organization, the higher the likelihood of encountering chaos; the larger the number of forces with different periodic patterns, the higher the likelihood of encountering chaos ${ }^{3}$.

Health care systems, hospitals and operating rooms are all organizations (albeit at differing levels) and are hence subject to chaos theory and, at times, actual chaos. As stressors on the system increase, the likelihood of problems increase. In the article by Ibrahim et al the issue of unexpected neurosurgical operating room cancellations is addressed - a situation that not infrequently can lead to chaos and frustration ${ }^{1}$. The authors adapt the A4R framework which has four primary expectations (or conditions), namely: 1. relevance, achieved by support for the process and criteria for decisions amongst all stakeholders; 2 . publicity (or transparency), satisfied by the effective communication of the results of the deliberation; 3 . challengeability (or revisability), accomplished through a fair appeals process; and, 4. oversight (or enforcement), ensuring all 
stakeholders that opportunities for improvement are available. According to the article, in the authors' neurosurgical operating room a priority list of cases is established the day before (by a designated surgeon who has been selected by the neurosurgical division) using criteria previously established by the members of the neurosurgical division (condition 1). This list of cases is provided to the neurosurgeons operating the next day (condition 2) and each individual surgeon has the opportunity to challenge the prioritization of their own cases (condition 3). This way, if for some unforeseen reason a neurosurgical case must be cancelled (lack of beds, broken equipment, etc.) on the day of surgery, then decisions need not be made at the last minute, using questionable criteria and by individuals who do not necessarily have the support of the affected surgeon. In other words the problem of neurosurgical case cancellations has been addressed in a proactive rather than a reactive way and some order has been established (rather than chaos).

The authors of the article indicate that the use of the A4R framework has improved the functionality of the neurosurgical operating room when it comes to case prioritization by creating a fair, equitable and ethically justifiable process that holds individuals accountable to the standard of reasonableness (and one that thereby mitigates conflicts). ${ }^{1}$ Though the article does not directly compare the situation pre and post A4R implementation, one has little reason to doubt the authors' conclusion. Indeed one could see the A4R framework applied to other elements of the neurosurgical frustration list presented.

And is there a role for the A4R framework beyond the operating room? If uncertainty and the potential for chaos exist in the operating room then surely they exit for a larger organization such as a hospital (of which the operating room is just a small part) or a health care system (of which a hospital is just a small part). What often frustrates stakeholders such as patients, physicians, nursing staff, allied health care workers and hospital administrators is the uncertainty as to whether any framework at all is being used to guide important decisions and mitigate chaos 6 . As Daniels and Sabin state: "Accountability for reasonableness is the idea that the rationales for important managed care plan decisions should not only be publicly available, but should also be those that 'fair-minded' people can agree are relevant to pursuing appropriate patient care under necessary resource constraints" ${ }^{2,7}$. Perhaps small gains made in the neurosurgical operating room can be used as a catalyst to affect change beyond.

\section{REFERENCES}

1. Ibrahim GM, Tymianski M, Bernstein M. Priority setting in neurosurgery as exemplified by an everyday challenge. Can J Neurol Sci. 2013;40(3):378-83.

2. Daniels N, Sabin J. The ethics of accountability in managed care reform. Health Aff (Millwood). 1998;17:50-64.

3. Thietart RA, Forgues B. Chaos theory and organization. Organization Science. 1995;6:19-31.

4. Lorenz EN. Deterministic nonperiodic flow. J Atmospheric Sci. 1963;20:130-41.

5. Lorenz EN. The mechanics of vacillation. J Atmospheric Sci. 1963; 20:448-64

6. Otten R, Chen T. Change, chaos, adaption: the effects of leadership on a work group. Creat Nurs. 2011;17:30-5.

7. Daniels N, Sabin JE. Making insurance coverage for new technologies reasonable and accountable. J Am Med Assoc. 1998;279:703-4.

\author{
Joseph F. Megyesi \\ London Health Sciences Centre \\ London, Ontario, Canada
}

